\title{
Measurement of the modulus and phase of the linear coupling coefficient by analysis of the transverse beam profile
}

\author{
P. F. Tavares, A. L. Xavier, Jr., Liu Lin, L. Jahnel, and R. H. Farias \\ Laboratório Nacional de Luz Síncrotron, Cx. Postal 6192, CEP 13083-970, Campinas, Brazil
}

(Received 19 June 1998; published 24 September 1998)

\begin{abstract}
We study the dynamics of transverse oscillations near the linear coupling resonance excited by a pair of skew quadrupoles at the Laboratório Nacional de Luz Síncrotron UVX electron storage ring through the analysis of the beam profile. Transverse coherent oscillations were excited with a fast kicker and the profile of the oscillating beam was observed by focusing visible synchrotron radiation from a bending magnet onto a fast charge-coupled device camera. Using a single resonance approximation, we calculated the border of the time-averaged transverse beam profile as a function of the complex coupling coefficient $\kappa$, which characterizes the distribution of coupling fields along the storage ring. A leastsquares fit of the calculated beam profile border to the experimentally obtained isointensity contours provided a new method to determine both the modulus and the phase of $\kappa$. The values obtained for the modulus are in good agreement with those from the conventional normal mode tune separation technique, and the values obtained for the phase of $\kappa$ agree with calculations based on the model lattice and the known skew quadrupole distribution. [S1098-4402(98)00016-0]
\end{abstract}

PACS numbers: 41.85.Ew, 29.20.Dh

\section{INTRODUCTION}

Coupling between the horizontal and vertical motion (betatron coupling) is widely recognized as an important performance limitation in storage rings used as synchrotron radiation sources or as colliders. Coupling produces betatron tune shifts and vertical dispersion, reduces the dynamic aperture [1], and increases the vertical emittance (with a corresponding decrease in light source brilliance or collider luminosity [2]). Clearly, an increased coupling may also be beneficial, e.g., providing enhanced Touschek lifetime for a low energy beam by decreasing the electron beam density. Linear coupling (i.e., coupling by linear fields) may be caused by tilted quadrupoles, vertical closed orbit deviations in sextupoles, and solenoidal fields in detectors, whereas nonlinear coupling may be produced by interaction with the electric fields of ions trapped in the electron beam [3] as well as by space charge or beam-beam forces [4]. Close to a resonant condition, the dynamics of linear coupling can be described by a single complex parameter: the coupling coefficient $\kappa$. The measurement of $|\kappa|$ is routinely done by measuring the separation between the oscillating frequencies of the normal modes of the coupled system [5]. Other techniques to measure coupling usually involve transversely exciting the beam and observing the resulting coherent oscillations. The perturbation can be a kick [6] or continuous wave excitation [7]. More recently, observations of coherent oscillations on a turn-by-turn basis at two different locations in the storage ring have allowed the experimental determination of both the modulus and the phase of $\kappa$ from a Hamiltonian description of the coupled dynamics [8-10].

In this work, we present an experimental study of the linear coupled transverse beam dynamics in the vicinity of the $\nu_{x}-\nu_{y}=3$ resonance excited by skew quadrupoles. We introduce a new experimental technique to obtain the parameters characterizing the strength of the resonance (namely the modulus and phase of the coupling coefficient). The method consists of observing the time evolution of the transverse beam profile for a few milliseconds (a short time compared to the synchrotron damping time, but a long time compared to the betatron oscillation period) after exciting the beam with a fast (few hundred nanoseconds) horizontal kick. The acquired image is a projection onto the $x y$ plane of the phase space density distribution function $\rho\left(x, x^{\prime}, y, y^{\prime}\right)$ integrated over a very large number of turns (but still small enough that the system may be considered Hamiltonian). Many of the geometric characteristics of the phase space orbits reveal themselves in this time-averaged profile, allowing the direct observation of several aspects of the phase space geometry close to the resonance and the experimental determination of the parameters describing the resonance strength. In the particular case of the linear coupling resonance (specifically, the difference resonance [11]), the phase space geometry is described by the complex coupling coefficient $\kappa$ and the averaged distribution presents an axis of symmetry rotated with respect to the symmetry plane of the machine, as well as distortions of its outermost contour. The rotation angle can be correlated with the real part of the coupling coefficient $\operatorname{Re}[\kappa]$, while the detailed geometry of the outer contour of the averaged beam profile strongly depends on its imaginary part $\operatorname{Im}[\kappa]$. The Hamiltonian formalism $[11,12]$ applied to the resonance region of interest provides a transverse map from which the beam profile contour can be extracted. The reverse process allows the determination of both $\operatorname{Re}[\kappa]$ and $\operatorname{Im}[\kappa]$ given the beam contour by a least-squares minimization. The procedure is applied 
to the experimentally obtained averaged contours which yields experimental estimates of the modulus and phase of $\kappa$.

A similar technique has already been used to explore the phase space close to the one-dimensional sextupoleexcited third-integer resonance and can, in principle, be used to study more complex resonance structures with a fairly simple and inexpensive experimental setup.

This paper is organized as follows: In Sec. II we briefly review the application of the Hamiltonian formalism to the study of the linear coupling resonance and derive geometrical properties of the integrated beam profile as a function of the coupling coefficient. Those results are used to establish an image analysis algorithm to determine experimentally the coupling coefficient. In Sec. III we describe the Laboratório Nacional de Luz Síncrotron (LNLS) UVX storage ring and the experimental setup. In Sec. IV we present the experimental results and compare them to those from other measurement methods and calculations. The conclusions are presented in Sec. V.

\section{OUTLINE OF THE THEORY}

The Hamiltonian that describes transverse electron motion close to a linear coupling resonance excited by a distribution of skew quadrupoles is given by [11]

$$
\begin{aligned}
H\left(x, x^{\prime}, y, y^{\prime}, s\right)= & \frac{1}{2} x^{\prime 2}+\frac{1}{2} y^{\prime 2}+\frac{1}{2} K_{q}(s) x^{2} \\
& -\frac{1}{2} K_{q}(s) y^{2}+K(s) x y,
\end{aligned}
$$

where $x$ and $y$ are the transverse electron coordinates, primes denote differentiation with respect to the longitudinal coordinate $s, K_{q}(s)$ describes the normal focusing quadrupoles, and

$$
K(s)=\frac{e c}{E} \frac{\partial B_{x}(s)}{\partial x}
$$

is the skew quadrupole strength. ${ }^{1}$ Here $E$ is the electron energy, $e$ is the electron charge, and $c$ is the velocity of light. The Hamiltonian (1) can be written in a suitable form for study by the method of variation of constants [12], whose skeleton is given by the perturbed solutions

$$
u(s)=\sqrt{2 a_{u} \beta_{u}(s)} \cos \left[\psi_{u}(s)+\Phi_{u}\right],
$$

where $u$ denotes either $x$ or $y$ planes. The functions $\beta_{u}(s)$ are the betatron functions (periodic functions over the ring circumference $L)$ and $\psi_{u}(s)$ are the corresponding betatron phases. The method looks for new equations for the new variables $a_{u}$ and $\Phi_{u}$. As a function of these new

\footnotetext{
${ }^{1}$ The function $K(s)$ is zero everywhere except inside the skew quadrupole, where it is assumed to be constant and equal to $K_{s}$.
}

variables, the coupling term in (1) is

$$
\begin{aligned}
H_{1}\left(a_{x}, a_{y}, \Phi_{x}, \Phi_{y}, s\right)= & 2 K(s) \sqrt{\beta_{x}(s) \beta_{y}(s) a_{x} a_{y}} \\
& \times \cos \left[\psi_{x}(s)+\Phi_{x}\right] \\
& \times \cos \left[\psi_{y}(s)+\Phi_{y}\right] .
\end{aligned}
$$

By changing the independent variable to $\varphi=2 \pi s / L$ and expanding the periodic Hamiltonian in Fourier series, we obtain

$$
\begin{aligned}
& \tilde{H}_{1}\left(a_{x}, a_{y}, \Phi_{x}, \Phi_{y}, \varphi\right) \\
& \quad=\sum_{q}\left|\kappa_{q}\right| \sqrt{a_{x} a_{y}} \cos \left[\Phi_{x}-\Phi_{y}+\Delta \varphi+\arg \left(\kappa_{q}\right)\right],
\end{aligned}
$$

where

$$
\begin{aligned}
\kappa_{q}=\frac{1}{2 \pi} \int_{0}^{L} & \sqrt{\beta_{x}(s) \beta_{y}(s)} K(s) \\
& \times \exp \left\{i\left[\psi_{x}(s)-\psi_{y}(s)-2 \pi s \Delta / L\right]\right\} d s,
\end{aligned}
$$

$\nu_{u}=\frac{\psi_{u}(L)}{2 \pi}$,

and $\Delta=\nu_{x}-\nu_{y}-q$. The new Hamiltonian (3) describes the particle motion close to the conveniently chosen difference resonance given by $\Delta$. It explicitly depends on the coupling coefficient $\kappa_{q}$, which is, in general, a complex quantity. We consider the isolated resonance $q=3$, i.e., we assume that we are close to the resonance condition $\nu_{x}-\nu_{y}-3 \approx 0$ and neglect all nonresonant terms in the Hamiltonian of Eq. (3). To simplify the notation we use $\kappa_{3}=\kappa$. In order to obtain the particle transverse motion, we apply another canonical transformation to the rotating system $\left(\tilde{a}_{u}, \tilde{\Phi}_{u}\right)$ generated by

$$
\begin{aligned}
G= & \tilde{a}_{x}\left[\Phi_{x}+\frac{1}{2} \Delta \varphi+\frac{1}{2} \arg (\kappa)\right] \\
& +\tilde{a}_{y}\left[\Phi_{y}-\frac{1}{2} \Delta \varphi-\frac{1}{2} \arg (\kappa)\right],
\end{aligned}
$$

in terms of which the new Hamiltonian is $\wp=$ $\tilde{H}_{1}+\partial G / \partial \varphi$ and the new variables are given by $a_{u}=\partial G / \partial \Phi_{u}$ and $\tilde{\Phi}_{u}=\partial G / \partial \tilde{a}_{u}$. This transformation eliminates the explicit dependence of the Hamiltonian on the orbital parameter $\varphi$. Finally, a transformation to $(w, v)$ defined by

$$
w=\sqrt{\tilde{a}_{x}} \exp \left(i \tilde{\Phi}_{x}\right), \quad v=\sqrt{\tilde{a}_{y}} \exp \left(i \tilde{\Phi}_{y}\right) y
$$

leads to the equations of motion

$$
\left(\begin{array}{c}
\ddot{w} \\
\ddot{v}
\end{array}\right)=\left[\begin{array}{cc}
i \Delta / 2 & i(\kappa) \\
i(\kappa) & -i \Delta / 2
\end{array}\right]\left[\begin{array}{l}
w \\
v
\end{array}\right] .
$$

Solutions to (6) are subjected to the choice of initial conditions. However, since we are only interested in the structure in phase space which emerges for a large number of turns, the exact initial conditions are not relevant and we take, for simplicity, $w(0)=w_{0}$ and $v(0)=0$. For 
these new variables, the determinant of the characteristic matrix in (6) gives the beating frequency

$$
\nu=\frac{1}{2} \sqrt{\left|\kappa_{q}\right|^{2}+\Delta^{2}}
$$

and, finally, the motion at the observation point is given by

$$
\begin{aligned}
\frac{x(\varphi)}{x_{0}} & =\cos \nu \varphi \cos \nu_{x x} \varphi-\frac{\Delta}{2 \nu} \sin \nu \varphi \sin \nu_{x x} \varphi \\
\frac{y(\varphi)}{x_{0}}= & -\sqrt{\frac{\beta_{y}}{\beta_{x}}} \frac{1}{2 \nu}\left[\operatorname{Im}(\kappa) \cos \nu_{y y} \varphi+\operatorname{Re}(\kappa) \sin \nu_{x x} \varphi\right] \\
& \times \sin \nu \varphi,
\end{aligned}
$$

with

$$
\nu_{x x}=\nu_{x}-\frac{\Delta}{2}, \quad \nu_{y y}=\nu_{y}+\frac{\Delta}{2} .
$$

In Eqs. (7) and (8), the motion is normalized according to the initial horizontal kick $x_{0}$, and $\beta_{y}$ and $\beta_{x}$ are the betatron function values at the observation point. The transverse map is obtained by noting that $\varphi$ is a multiple of $2 \pi$ so that $[x(2 \pi j), y(2 \pi j)]$ represents the transverse beam position vector of the $j$ th beam passage at the observation point. Note that we have taken the observation point as the origin for the integral calculation of $\kappa$, Eq. (4). The horizontal and vertical oscillations can be factored into a fast oscillation (determined by $\nu_{x}$ and $\nu_{y}$ ) and a slow amplitude (determined by $\nu$ ) modulation term. The slow modulation is easily seen from the expression for the amplitudes

$$
\begin{gathered}
a_{x}(\varphi)=\frac{\left|w_{0}\right|^{2}}{4 \nu^{2}}\left(\Delta^{2}+|\kappa|^{2} \cos ^{2} \nu \varphi\right), \\
a_{y}(\varphi)=\frac{\left|w_{0}\right|^{2}}{4 \nu^{2}}|\kappa|^{2} \sin ^{2} \nu \varphi .
\end{gathered}
$$

Figure 1 shows two examples of two-dimensional integrated beam profiles as given by expressions (7) and (8). If the coupling coefficient is real [as in Fig. 1(b)], the border of the integrated profile is a parallelogram rotated by an angle $\theta$ with respect to the machine horizontal axis of symmetry with $\theta$ given by ${ }^{2}$

$$
\tan \theta=\sqrt{\frac{\beta_{y}}{\beta_{x}}} \frac{\Omega}{1+\sqrt{1+\Omega^{2}}}, \quad \Omega=\frac{\operatorname{Re}(\kappa)}{\Delta} .
$$

The expression above is found by considering the time evolution of the oscillation amplitudes, Eqs. (9) and (10), and noting that the maximum vertical amplitude takes place at the point of minimum horizontal amplitude. Also, the maximum horizontal amplitude occurs at zero vertical

\footnotetext{
${ }^{2}$ If $\operatorname{Im}(\kappa) \neq 0$, the integrated profile becomes distorted and is no longer a parallelogram. However, another parallelogram also rotated by an angle given by Eq. (11) can still be circumscribed to the image.
}
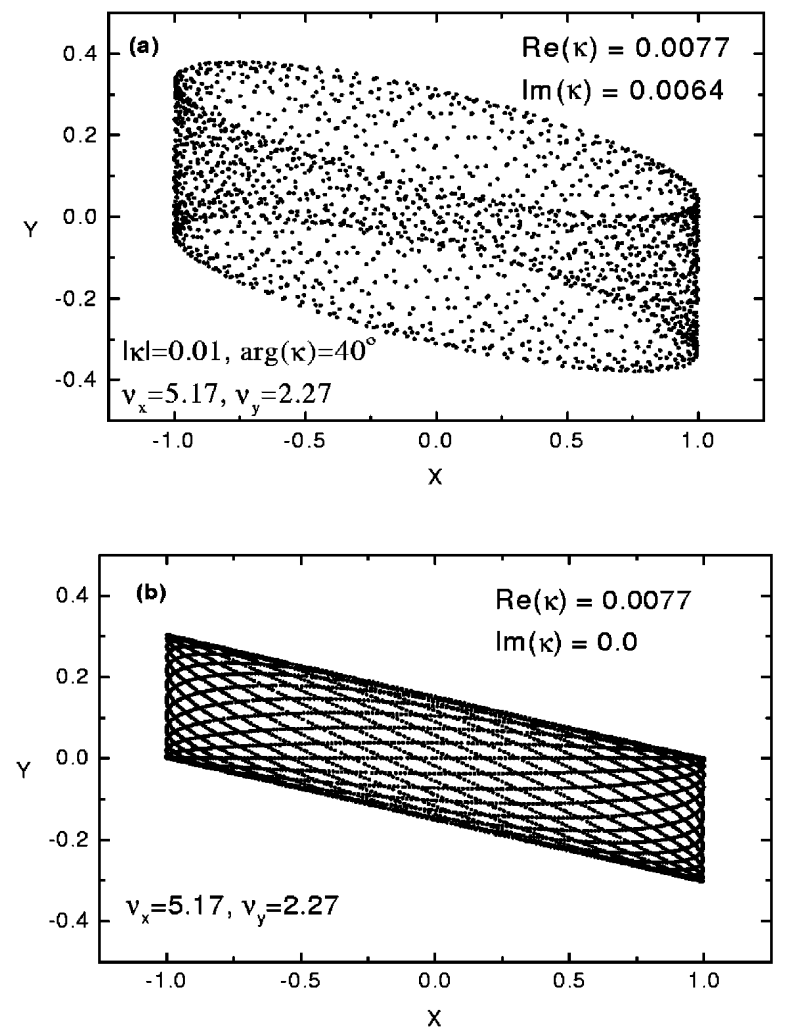

FIG. 1. Examples of integrated beam transverse profiles that result from Eqs. (7) and (8).

amplitude. This gives us two points of the parallelogram and (by symmetry) the rotation angle. In the limit $\beta_{x} \rightarrow$ $\beta_{y}$, relation (11) is equivalent to the expression found by Chao and Lee [13] for the slope of the stationary transverse electron density distribution determined by the equilibrium between the excitation of the oscillations due to the discrete nature of synchrotron radiation emission (quantum excitation) and the damping due to the average radiation emission rate under the action of the coupling fields of a skew quadrupole. The same expression is obtained here in a different context in which coherent undamped time-averaged oscillations are considered.

\section{A. Extracting the beam profile border}

Here we show how to obtain the border of the integrated transverse profile. In a certain sense, our aim is to find a reference system simpler than the ordinary $(x, y, \varphi)$ which allows us to write the coupling coefficient $\kappa$ as a function of the profile border. To this aim we define

$$
\begin{gathered}
g_{11}=\cos \nu \varphi, \quad g_{12}=-\frac{\Delta}{2 \nu} \sin \nu \varphi, \\
g_{21}=-\frac{1}{2 \nu} \sqrt{\frac{\beta_{y}}{\beta_{x}}} k_{2} \sin \nu \varphi, \\
g_{22}=-\frac{1}{2 \nu} \sqrt{\frac{\beta_{y}}{\beta_{x}}} k_{1} \sin \nu \varphi,
\end{gathered}
$$


where $k_{1}=\operatorname{Re}|\kappa|$ and $k_{2}=\operatorname{Im}|\kappa|$. Here again, $\varphi(j)=$ $2 \pi j$ with $j$ a positive integer. The position vector $\vec{R}=$ $(x, y)$ describes ellipses whose parameters vary slowly according to $\nu$. Defining $\bar{\nu}=\left(\nu_{x}+\nu_{y}\right) / 2$, we can write the map (7) and (8) in the form

$$
\left(\begin{array}{l}
x \\
y
\end{array}\right)=(-1)^{j}\left(\begin{array}{ll}
g_{11} & g_{12} \\
g_{21} & g_{22}
\end{array}\right)\left(\begin{array}{c}
\cos \bar{\nu} \varphi(j) \\
\sin \bar{\nu} \varphi(j)
\end{array}\right) .
$$

There are, therefore, two regimes of motion as already discussed: a slow one given by $\nu$ and a fast one determined by $\bar{\nu}$. The square of the distance from the center is

$$
\begin{aligned}
R^{2}= & \frac{1}{2}\left(g_{11}^{2}+g_{12}^{2}+g_{21}^{2}+g_{22}^{2}\right) \\
& +\frac{1}{2}\left(g_{11}^{2}+g_{21}^{2}-g_{12}^{2}-g_{22}^{2}\right) \cos 2 \Psi \\
& +\left(g_{11} g_{12}+g_{21} g_{22}\right) \sin 2 \Psi,
\end{aligned}
$$

with $\Psi=\bar{\nu} \varphi(j)$. Points at the border of (12) obey the relation

$$
\frac{\partial R^{2}}{\partial \varphi}=0
$$

together with the constraint condition

$$
\tan \gamma=\frac{y}{x}=\phi
$$

where $\gamma$ is the polar coordinate of the point and $\phi$ is fixed. This means that for each value of $\gamma(0 \leq \gamma \leq \pi$, because of the symmetry) we must find the solutions which satisfy Eq. (14). The dependence on $\Psi$ can be eliminated from $R^{2}$ so that the new map is given in terms of the slow motion only. We note that

$$
\tan \gamma=\frac{g_{21}+g_{21} \cos 2 \Psi+g_{22} \sin 2 \Psi}{g_{11}+g_{11} \cos 2 \Psi+g_{12} \sin 2 \Psi},
$$

and

$$
\beta=\frac{g_{22} \cos \gamma-g_{12} \sin \gamma}{g_{11} \sin \gamma-g_{21} \cos \gamma},
$$

so that

$$
\cos 2 \Psi=\frac{\beta^{2}-1}{\beta^{2}+1}, \quad \sin 2 \Psi=\frac{2 \beta}{\beta^{2}+1} .
$$

It is convenient now to introduce new terms,

$$
\begin{gathered}
m=\frac{-\Delta}{2 \nu}, \\
l_{1}=-\frac{1}{2 \nu} \sqrt{\frac{\beta_{y}}{\beta x}} k_{1}, \quad l_{2}=-\frac{1}{2 \nu} \sqrt{\frac{\beta_{y}}{\beta x}} k_{2},
\end{gathered}
$$

and we can write Eq. (13) as

$$
R^{2}=\frac{\left[\left(\phi^{2}+1\right) l_{2}^{2}+2 l_{1} l_{2}\right] \beta^{4}+\left(\phi^{2}+1\right) l_{1}^{2} \beta^{2}+2 \phi^{2} l_{1} l_{2} \beta^{3}}{\left(1+\beta^{2}\right)\left[\left(l_{1}+\beta l_{2}-m \phi\right)^{2}+\beta^{2} \phi^{2}\right]}
$$

It is possible, however, to further simplify relation (15) by noting that $y=R \sin \gamma$ and defining $p=\beta^{-1}$. We get finally

$$
R^{2}=\frac{\left(\phi^{2}+1\right)\left(l_{2}+l_{1} p\right)^{2}}{\left(p^{2}+1\right)\left\{\left[\left(l_{1}-m \phi\right) p+l_{2}\right]^{2}+\phi^{2}\right\}},
$$

and our transformation of reference system is now completed: $(x, y, \varphi) \rightarrow(R, \phi, p)$. Equation (14) is simply $\partial R^{2} / \partial p=0$, which leads us to find the roots of a fourth order polynomial in $p$ as a function of $\phi$

$$
\begin{aligned}
\phi\left(\phi l_{1}+m l_{2}^{2}\right)+l_{2}\left[\phi l_{1} m-l_{2}^{2}-\phi^{2}\left(1+m^{2}\right)\right] p+ \\
3 l_{2}^{2}\left(\phi m-l_{1}\right) p^{2}+l_{2}\left(3 l_{1}-2 \phi m\right)\left(\phi m-l_{1}\right) p^{3}- \\
l_{1}\left(\phi m-l_{1}\right)^{2} p^{4}=0 .
\end{aligned}
$$

(In fact, the polynomial is of fifth order with one of the roots $R=0$.) All of the relevant solutions belong to the family branch starting at $p=0$ for $\phi=0$. An iterative procedure can generate solutions for other values of $\phi$ and, in this way, the whole border is obtained. Figure 2 shows a (normalized) plot of $R$ in terms of $\gamma$ for the same parameters of Fig. 1(a).

\section{B. Getting $\kappa$ from the profile border analysis}

In order to experimentally obtain the beam profile border, we must determine the isointensity curves of the transverse beam profile (contour plots). Then we choose the contour that best represents the border and apply a least-squares fit based on Eq. (16) subjected to the roots

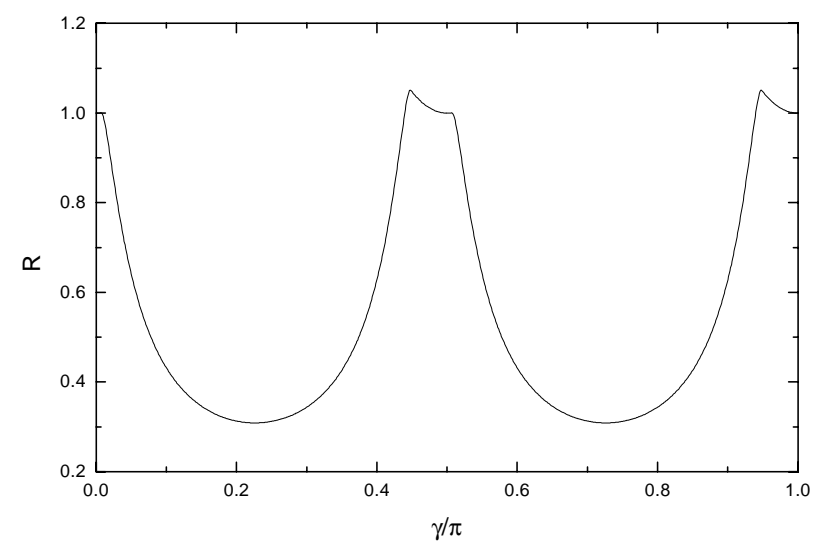

FIG. 2. Calculated profile contour $R(\gamma)$ for the same parameters as Fig. 1(a). 
of (17) in order to extract the best value of $\kappa$. Here we sketch some details of the fitting procedure.

Given a certain experimental contour of $N$ points in polar coordinates $R^{\star}\left(\phi_{i}\right)\left(0 \leq \phi_{i} \leq 2 \pi\right)$, we construct a figure-of-merit function

$$
\begin{aligned}
\chi^{2}\left(k_{1}, k_{2}, z\right) & =\sum_{i=1}^{N} \frac{1}{\sigma^{2}}\left[R^{\star}\left(\phi_{i}\right)-R\left(k_{1}, k_{2}, z\right)\right]^{2}, \\
z & =\frac{\beta_{y}}{\beta_{x}},
\end{aligned}
$$

where $R\left(k_{1}, k_{2}, z\right)$ is given by (16) subjected to the solution of (17) and $\sigma^{2}$ is the variance associated with the measurement of a given contour. This variance depends on several details such as camera resolution, pixel size, and the area of the image matrix. Also, the beam is not a point in the transverse space but rather a Gaussian distribution with a characteristic spread. Here we will take $\sigma^{2}$ as simply the variance associated with the basic cell of the image matrix (typically $10^{-4}$ ). Moreover, in the form (18) the ratio $z$ at the observation point is one of the fitted parameters. The minimum of (16) is located in the direction of the vector $-\vec{\nabla} \chi^{2}$, and it is straightforward to iteratively correct an initial parameter guess until a convenient minimum of (18) is reached [14].

In order to test the validity of the presented method, we generate a "surrogate" image by counting the number of points generated by the map (7) and (8) inside each cell of the image matrix. The resulting image histogram (whose parameter set is known) can then be analyzed and its parameter set obtained. This procedure also enables us to determine which contour (or set of contours) must be taken in order to obtain a good fit. In general, if the image histogram is normalized (background level at 0 and maximum intensity 1), the best contours for the fitting occur around 0.1 .

\section{EXPERIMENTAL SETUP}

\section{A. The LNLS UVX storage ring}

The LNLS UVX electron storage ring [15] (Fig. 3) is a $1.37 \mathrm{GeV}$ machine injected from a $120 \mathrm{MeV}$ linac.
The lattice is a sixfold symmetric double-bend achromat with alternating long (nondispersive) and short (dispersive) straight sections and a total length of $93.21 \mathrm{~m}$ (3.2 MHz revolution frequency). A pair of skew quadrupoles (AQS05A and AQS05B) is installed in one of the long straight sections and is used to drive the coupling resonance. One of the injection kickers was used to excite a coherent betatron oscillation, and the subsequent evolution of the transverse beam profile was observed with a synchrotron radiation monitor. The beam energy was $600 \mathrm{MeV}$ in order to have a sufficiently long damping time $(90 \mathrm{~ms})$ with respect to the image integration time (8.6 ms).

We used two different modes of operation during the experiments: the standard optics and the low vertical beta optics (Fig. 4). In the standard (sixfold symmetric) operation mode, the horizontal and vertical betatron phase advances from one skew quadrupole to the other are rather small (since both $\beta_{x}$ and $\beta_{y}$ are large along the straight section). Also, since they are symmetrically installed with respect to the center of the straight section, the betatron functions at both quadrupoles are equal so that $|\kappa|$ and $\arg (\kappa)$ produced by powering either quadrupole are essentially the same. Therefore, in the normal operating mode, our measurements were done with both skew quadrupoles powered in series. The low vertical beta optics was implemented in the straight section containing the pair of skew quadrupoles (breaking the sixfold symmetry of the lattice) in order to provide a large vertical betatron phase advance from one skew quadrupole to the other (while keeping the horizontal phase advance small) and thus allow the phase of the coupling coefficient to be varied by powering each skew quadrupole separately. We also measured the betatron functions at the focusing quadrupoles in the low-beta section in order to check the model optics of this mode. The betatron function measurements agreed with theoretical predictions to within $20 \%$.

\section{B. The optical beam profile monitor}

The optical characterization bench (Fig. 5) uses visible radiation from a low dispersion $\left(4^{\circ}\right)$ bending magnet port.

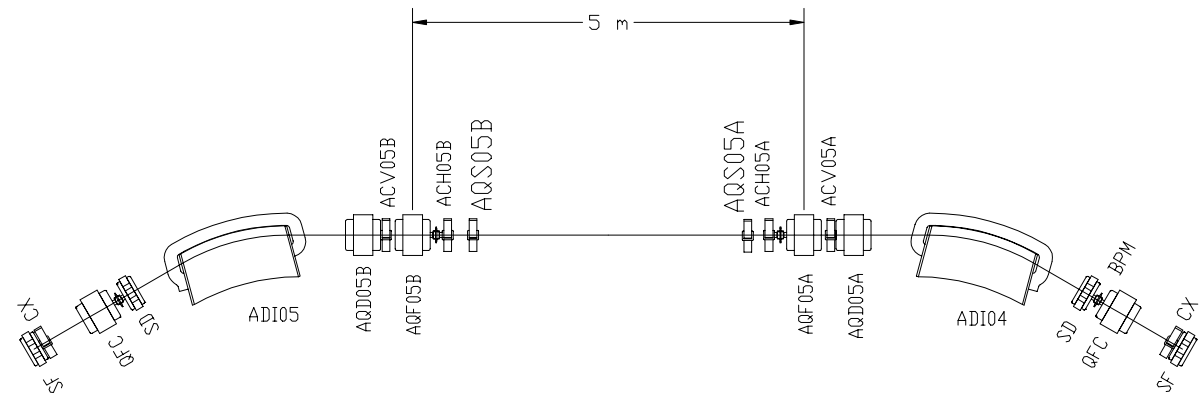

FIG. 3. Schematic drawing of a superperiod of the LNLS UVX electron storage ring where one can see the skew quadrupoles used in the experiment (AQS05A and AQS05B) and both focusing and defocusing normal quadrupole magnets (AQF05A, AQF05B, AQD05A, AQD05B). 

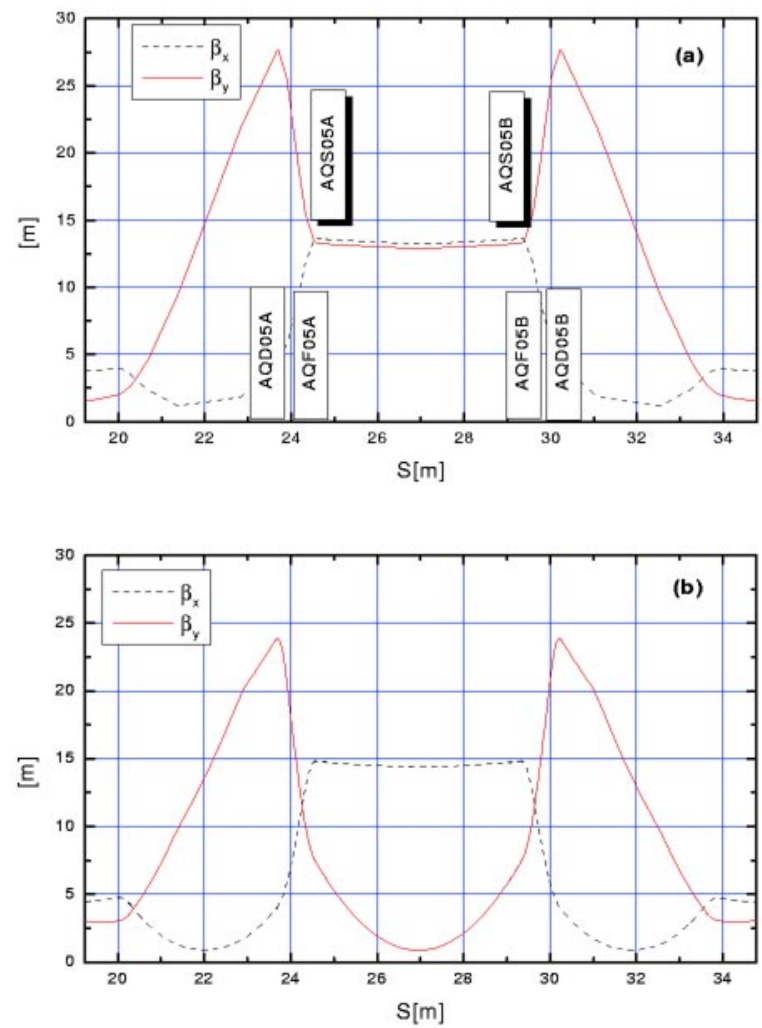

FIG. 4. (Color) Betatron functions in the standard optics mode (a) and the low-beta optics mode (b). The positions of the skew quadrupoles are indicated.

The high energy synchrotron radiation power is absorbed by a sapphire filter connected to a water cooled copper mask. The visible portion of the spectrum is extracted to the atmosphere by means of another sapphire window and guided to the characterization line by means of two first-surface mirrors with $86^{\circ}$ and $90^{\circ}$ deflection angles. Two plane-convex lenses form an image of the transverse profile of the beam on a CCD sensor. A slit installed in front of the optical system defines the horizontal and vertical apertures, thus controlling diffraction and

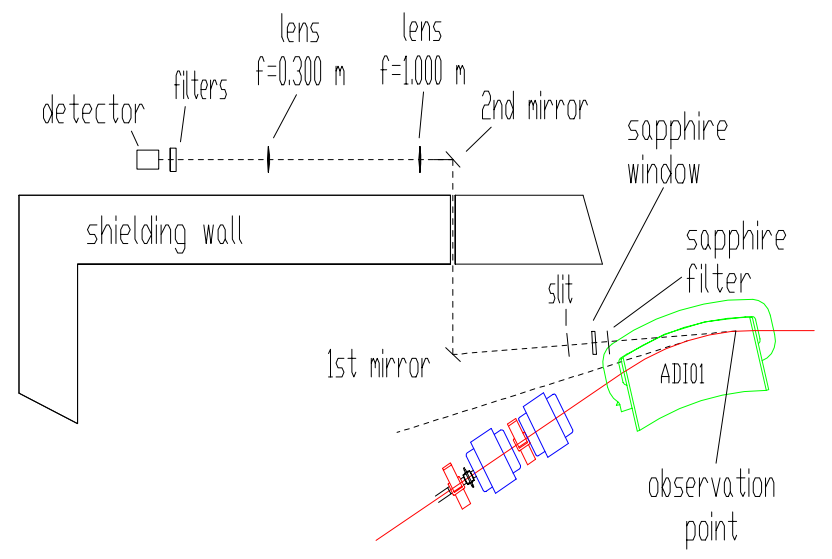

FIG. 5. (Color) View of the optical beam profile monitor. geometric curvature effects. The visible radiation is monochromatized by means of a $10 \mathrm{~nm}$ bandpass filter in order to reduce chromatic aberrations, and a pair of polaroids is used to control the light intensity on the CCD, thus avoiding saturation. The polaroids are installed close to the CCD sensor in order to minimize errors due to surface irregularities. The first mirror, as well as the polaroid positions, can be remotely adjusted.

The CCD sensor is an EEV CAM17-46E camera capable of producing nonstandard video signals which provide up to a $116 \mathrm{~Hz}$ frame rate. This is important in order to guarantee that the total frame integration time is small with respect to the synchrotron damping time at the energies of interest. The CCD spatial resolution is $15 \mu \mathrm{m}$, and the camera output signal is digitized with a Matrox Pulsar frame grabber board which provides 8 bit amplitude resolution and the possibility of using an external trigger signal to start frame acquisition. This feature was used in order to synchronize the frame acquisition with the excitation of the beam by means of the kicker magnet.

\section{RESULTS AND DISCUSSION}

Figure 6 shows an example of the integrated transverse profile of a kicked beam. Both pictures were taken in the normal mode (standard optics) with the skew quadrupoles powered in series. Note the inversion of the slope of the figure when we move the tunes from one side of the resonance to the other (invert the sign of $\Delta$ ).

\section{A. Standard optics}

Figure 7 shows the measured value of the variation of $|\kappa|$ as a function of quadrupole strength $K$ as given by the analysis of the beam image and by the conventional tune separation technique. We plot the variation of $|\kappa|$ rather than the coupling coefficient itself in order to eliminate offsets due to the effect of remanent fields in the skew quadrupoles. The normal mode separation is measured by scanning the vertical tune toward the horizontal tune and measuring their minimum distance of approximation. The slope of the curves agrees with the theoretical result $(|\kappa|=0.43 K)$ to within $30 \%$, which

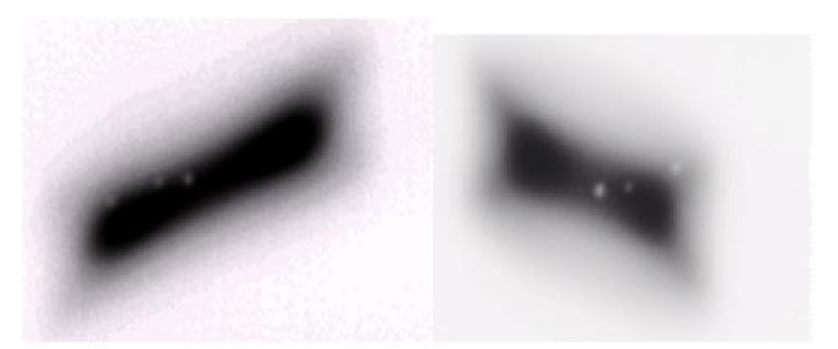

FIG. 6. Examples of integrated kicked beam profile images. The betatron tunes are $\nu_{x}=5.21, \nu_{y}=2.17$ (left) and $\nu_{x}=$ $5.14, \nu_{y}=2.17$ (right). 


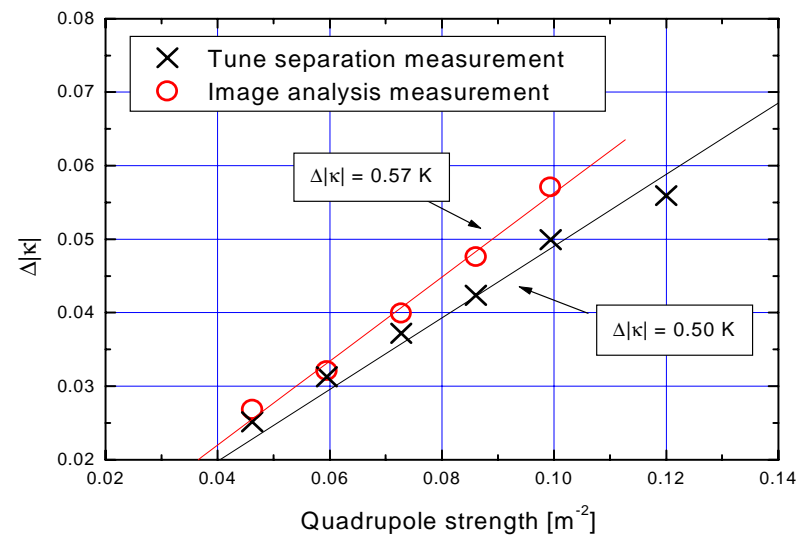

FIG. 7. (Color) Measurement of the variation of $|\kappa|$ as a function of quadrupole magnet strength $K$ in the standard (sixfold symmetric) optics. The betatron tunes are $\nu_{x}=$ 5.243 and $\nu_{y}=2.196$, and the skew quadrupoles are powered in series. Two data sets are shown corresponding to the measurement by analysis of the kicked beam image (circles) and measurement of the tune separation of the normal modes (crosses).

may be considered reasonable since the accuracy of the betatron function measurements (on which the calculated slope depends) is of the order of $20 \%$. The agreement with the results from the tune separation measurements is better (13\%). Figure 8 shows the measurements of the phase of the coupling coefficient as a function of quadrupole excitation for both positive and negative values of $K$. Figure 9 shows the measured change in phase due to the quadrupole strength sign inversion. As expected from theory, the phase jump is close to $180^{\circ}$.

\section{B. Low-beta optics}

In the low-beta optics, each skew quadrupole was powered independently; Figs. 10 and 11 show the variation of $|\kappa|$ as a function of either quadrupole magnet strength. As expected, the modulus of the coupling coefficient is

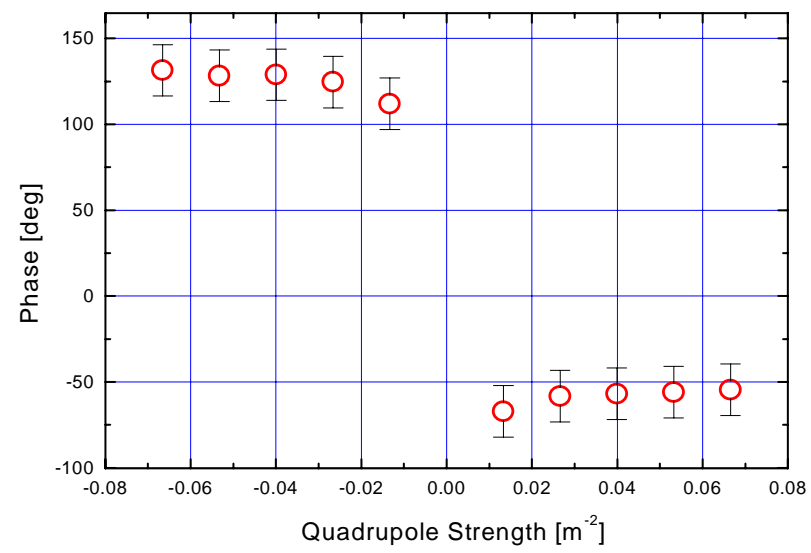

FIG. 8. (Color) Measurement of the phases of the coupling coefficient as a function of the quadrupole magnet strength in the standard optics.

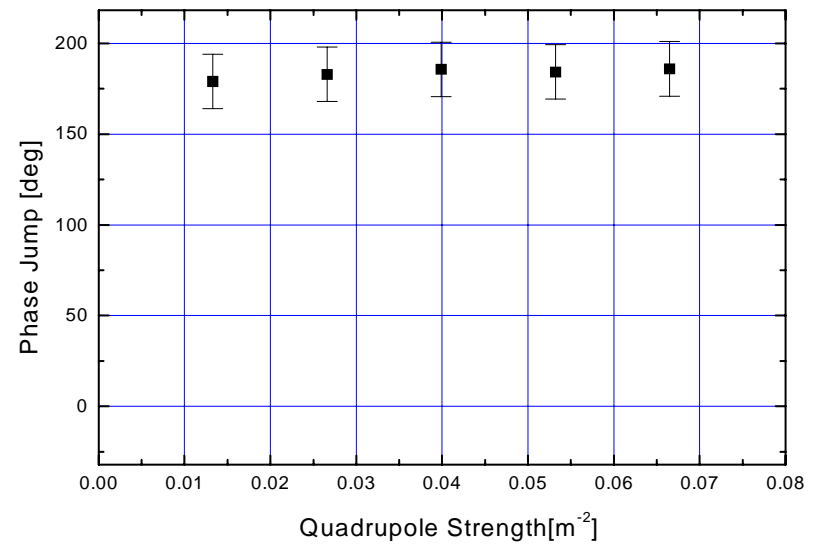

FIG. 9. (Color) Change in phase due to the inversion of sign of the quadrupole strength as a function of $K$.

largely insensitive to the betatron phase advance from one quadrupole to the next. The slope of both curves agrees with the theoretical estimate $(|\kappa|=0.13 K)$ based on the model optics to within $15 \%$.

Figure 12 shows the measured change in the phase of the coupling coefficient as we power skew quadrupole $A$ instead of skew quadrupole $B$ as a function of quadrupole strength. This change in phase reflects the large vertical betatron phase advance between the two skew quadrupoles and can be computed once we know the betatron functions at the center of the straight section by

$$
\delta \psi=2\left[\arctan \left(\frac{s}{\beta_{0 x}}\right)-\arctan \left(\frac{s}{\beta_{0 y}}\right)\right]-2 \Delta \frac{s}{L},
$$

where $s$ is the distance from the center of the straight section to either skew quadrupole and $\beta_{0 x}$ and $\beta_{0 y}$ are the betatron functions at the center of the section, which are estimated from measurements at the nearby focusing quadrupoles. The resulting calculated change in the phase of the coupling coefficient is $114^{\circ}$, which is about $15 \%$ above the experimentally determined values.

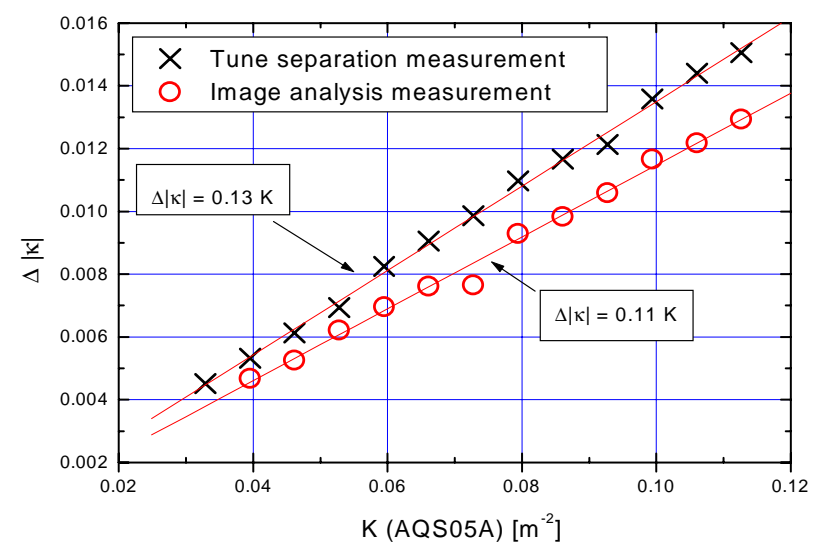

FIG. 10. (Color) Measurement of the variation of $|\kappa|$ as a function of quadrupole magnet strength in the low-beta optics. Only skew quadrupole $A$ is powered. 


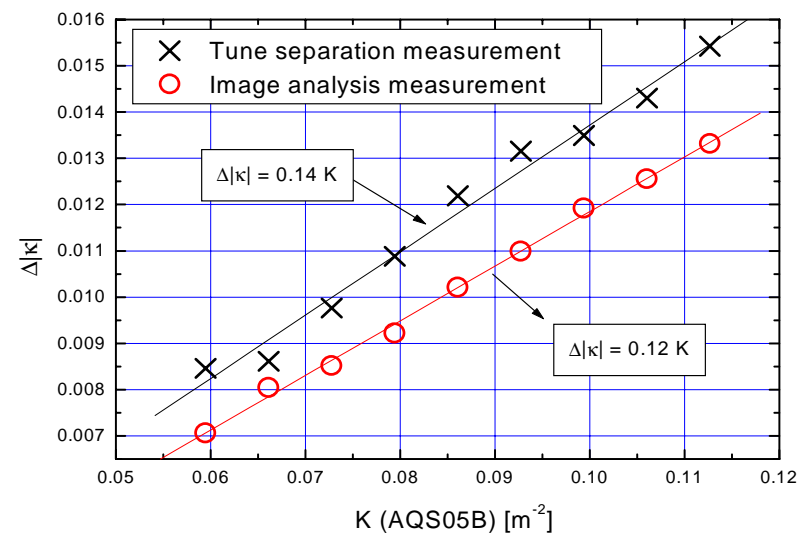

FIG. 11. (Color) Measurement of the variation of $|\kappa|$ as a function of quadrupole magnet strength in the low-beta optics. Only skew quadrupole $B$ is powered.

\section{Method limitations}

We now discuss some limitations of the profile analysis technique for measuring the coupling coefficient and discuss factors that influence the accuracy of the previous results. We estimate the error bars in the coupling coefficient modulus measurements to be \pm 0.002 in the case of the normal mode separation method. This is given by the tune measurement resolution. In order to estimate the error bars in the coupling measurements via image analysis, we have applied the image analysis algorithm to simulated profiles and calculated the spread in the resulting values of the coupling coefficient as we choose different isointensity contours for the fitting procedure. This gives \pm 0.001 for the measurement of the modulus of the coupling coefficient. These simulations do not take into account the fact that the theoretical analysis in Sec. II effectively assumes a single particle beam, which means that the beam size must be small with respect to the amplitude of the coherent oscillation. If this is not the

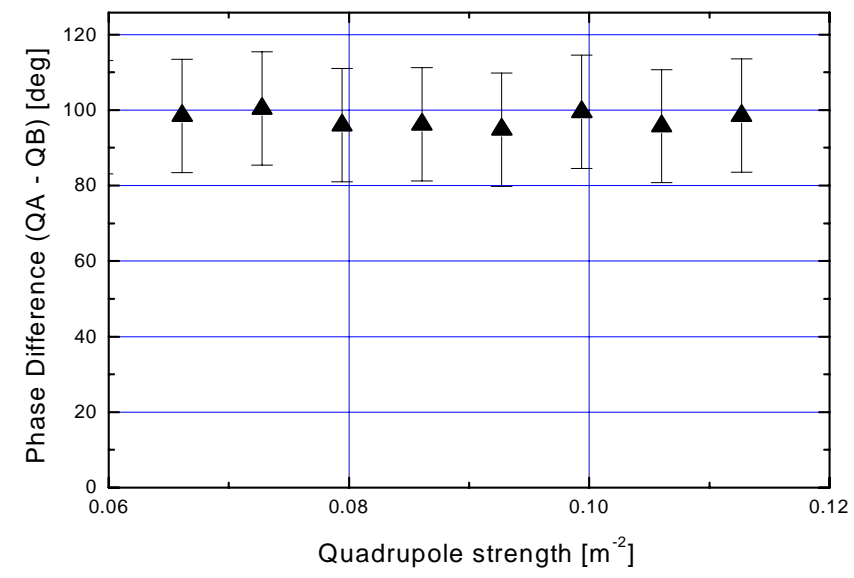

FIG. 12. (Color) Change in the phase of the coupling coefficient as we power quadrupole $A$ instead of quadrupole $B$ as a function of quadrupole strength in the low-beta optics. case, the beam profile smears out and the determination of $\operatorname{Im}(\kappa)$ (which is more critically dependent on the shape of the profile than on its orientation) becomes less precise. Therefore the kicker strength must be chosen so that the oscillation amplitude is large compared to the beam size, yet not so large that nonlinearities become important. Also, this implies that the skew quadrupole strength must be small since, on the contrary, large beam sizes will be produced.

The error bars in the phase measurements have been determined by simulation to be of the order of $15^{\circ}$ for the coupling phases we have measured. However, the uncertainty increases considerably for coupling phases close to zero. This happens because, for small coupling phases, large variations in phase cause comparatively little change to the kicked beam profile.

Finally, the method is limited by the validity of the isolated resonance approximation, i.e., for the tune region where the analytical map (7) and (8) correctly represents the motion. This is not a limitation in the sense that the very significance of the coupling coefficient $\kappa$ is also restricted to the region in tune space where the isolated resonance approach is valid.

The method presented here is quite cheap to implement and the hardware is simpler than the turn-by-turn beam position monitor (BPM) system used in [8]. Of course, a pair of turn-by-turn BPMs provides much more detailed information on the geometry of phase space than the averaged beam profile, which gives only a projection of phase space onto the $x y$ plane.

\section{CONCLUSION}

We have shown that the analysis of the transverse profile of a kicked beam integrated over a time short with respect to the damping time can provide useful information on the properties of the phase space geometry. In particular, in the vicinity of a coupling resonance, Hamiltonian perturbation theory was used to set up an image analysis algorithm to measure both the modulus and phase of the coupling coefficient $\kappa$. The results for $|\kappa|$ are in good agreement with theoretical estimates as well as with the results of a more conventional measuring technique. The results for the change in the phase of $\kappa$ due to a change in position of the source of coupling agree well with calculations.

\section{ACKNOWLEDGMENTS}

This work has been partially supported by FAPESP (Fundação de Amparo à Pesquisa do Estado de São Paulo) under Contracts No. 97/12948-4 and No. 95/9746-5.

[1] G. Ripken and F. Willeke, DESY Report No. 90-001, 1990. 
[2] G. Guignard, CERN Report No. ISR-B0M/77-43, 1977.

[3] P.F. Tavares, Report No. CERN-PS/92-54 (LP), 1992.

[4] B. W. Montague, Nucl. Instrum. Methods 187, 335 (1981).

[5] P. J. Bryant and K. Johnsen, The Principles of Circular Accelerators and Storage Rings (Cambridge University, Cambridge, England, 1993).

[6] P. J. Bryant and G. Guignard, CERN Report No. ISR-MA/ 75-42, 1975.

[7] J.P. Koutchouk, in Proceedings of the 11th International Conference on High Energy Accelerators, Geneva, 1980 (Birkhauser-Verlag, Basel, Switzerland, 1980).

[8] J. Y. Liu, M. Ball, B. Brabson, J. Budnick, D. D. Caussyn, V. Derenchuk, G. East, M. Ellison, D. Friesel, B. Hamilton, H. Huang, W. P. Jones, S. Y. Lee, D. Li, K. Y. Ng, A. Riabko, T. Sloan, and Y. Wang, Phys. Rev. E 49, 2347 (1994).

[9] D. D. Caussyn, M. Ball, B. Brabson, J. Collins, S. A. Curtis, V. Derenchuk, D. DuPlantis, G. East, M. Ellison, T. Ellison, D. Friesel, B. Hamilton, W. P. Jones, W. Lamble, S. Y. Lee, D. Li, M. G. Minty, T. Sloan, G. Xu, A. W. Chao, K. Y. Ng, and S. Tepikian, Phys. Rev. A 46 , 7942 (1992).
[10] M. Ellison, M. Ball, B. Brabson, J. Budnick, D. D. Caussyn, A.W. Chao, J. Collins, V. Derenchuk, S. Dutt, G. East, T. Ellison, D. Friesel, B. Hamilton, H. Huang, W. P. Jones, S. Y. Lee, D. Li, M. G. Minty, G. Nagaitsev, K. Y. Ng, X. Pei, T. Sloan, M. Syphers, L. Teng, Y. Wang, Y.T. Yan, and P.L. Zhang, Report No. FERMILAB-Conf-93/174, 1993.

[11] H. Wiedemann, Particle Accelerator Physics II (SpringerVerlag, Berlin, 1995).

[12] G. Guignard, Report No. CERN 78-11, 1978.

[13] A. Chao and M. Lee, J. Appl. Phys. 47, 4453 (1976).

[14] P. R. Bevington, Data Reduction and Error Analysis for the Physical Sciences (McGraw-Hill, New York, 1969).

[15] A. R. D. Rodrigues, R.H. A. Farias, M. J. Ferreira, G. S. Franco, L. C. Jahnel, Liu Lin, A. C. Lira, R. T. Neuenschwander, C. Pardine, F. Rafael, A. Rosa, C. Scorzato, C.E. T. Gonalves da Silva, A. Romeu da Silva, P.F. Tavares, D. Wisnivesky, and A. Craievich, in Proceedings of the 1997 Particle Accelerator Conference, Vancouver, Canada (to be published). 